\title{
Future health care applications resulting from progress in the neurosciences: the significance of neural plasticity research
}

\author{
A.C. Gelijns ${ }^{1}$, P.J. Graaff ${ }^{2}$, F.H. Lopes da Silva ${ }^{3}$ and \\ W.H. Gispen ${ }^{4}$ \\ ${ }^{1}$ Institute of Medicine, National Academy of Sciences, Washington, DC, \\ U.S.A., ${ }^{2}$ Project on Future Health Care Technology, Health Council, The \\ Hague, The Netherlands; ${ }^{3}$ Department of General Zoology, University of \\ Amsterdam, Amsterdam, The Netherlands; and ${ }^{4}$ Division of Molecular \\ Neurobiology, University of Utrecht, Utrecht, The Netherlands
}

Accepted 24 September 1987

\section{Summary}

Neurological, communicative and behavioral disorders afflict a significant part of the population in industrialized countries, and these disorders can be expected to gain in importance in the coming decades. In a considerable number of these disorders impairments in plasticity, i.e. deficiencies in the adaptive and regenerative capacity of the nervous system, play a central role. This paper considers the possibilities and probabilities of future improvements in treatment and prevention for a number of nervous system disorders following from neural plasticity research. Subsequently, it discusses the overall health policy implications of progress in the area of neural plasticity research and development in particular, and the neurosciences in general.

Neural plasticity; Nervous system disorder; Trends in neurosciences; Technology and health policy; Alzheimer's disease; Parkinson's disease; Injury of the nervous system

Address for correspondence: A.C. Gelijns, International Fellow, Council on Health Care Technology, Institute of Medicine, National Academy of Sciences, 2101 Constitution Avenue, Washington, DC 20418, U.S.A. 


\section{Introduction}

The quest for knowledge about the human nervous system, especially the inquiry into the structure and functioning of man's brain, is one of the most complex challenges in science. Neurosciences is the multidisciplinary scientific field, which investigates the normal and abnormal development, organization, and operation of the nervous system. At present the basic mechanisms underlying nervous system disorders are largely unknown. However, the field is in great motion as increasingly advanced monodisciplinary technology and know-how is applied to what in essence are multidisciplinary problems. Examples of developments, that are evolving towards multidisciplinarity, are the application of recombinant-DNA technology to the understanding of age-related diseases such as senile dementia; the application of non-invasive imaging techniques to provide anatomical and functional information about specific brain regions; the application of micro-electronics and new materials to develop sophisticated neurological prosthetics; or the application of information-processing paradigms to examine psychological and cognitive processes. In the coming years, the continuous application of advanced technology and know-how, and the intergration of monodisciplinary approaches into multidisciplinary programs can be expected to result in new and improved preventive and treatment measures for neurological, communicative, and behavioral disorders.

At present these nervous system disorders - which include, among others, dementia, affective disorders, impaired vision, multiple sclerosis and nervous system trauma - afflict a significant part of the population in industrialized countries. Most of them are chronic and dehumanizing diseases, which lead to an immense amount of personal suffering, as well as raising issues for society such as the need for and cost of care and support. For example, in several countries the inhabitants of nursing homes consist predominantly of patients with dementia and stroke. Furthermore, as many of the neurodegenerative and communicative disorders are correlated with age, their prevalence can be projected to rise in future due to aging population structures in most countries.

Thus, emerging and future technological applications of neurosciences research may well have large implications for the organization and delivery of health care and subsequently perhaps for mortality and morbidity patterns of nervous system disorders. In a field as culturally sensitive as that involving the functioning of the human brain with its relation to quintessential issues such as mind, nature of consciousness, autonomous expression of will, or intelligence, certain technological developments may also raise social and ethical issues.

In recognition of the high scientific and technological potential of the field on the one hand, and the potentially large implications of new technological applications for our health sectors on the other, a prospective assessment of possible new developments in the neurosciences has been recently undertaken in the Netherlands [1]. The study was part of an overall project analyzing future health care technology, described earlier on in this Journal. In this paper some findings are presented concerning a specific area of research within the neurosciences, i.e. 
progress in research on neural plasticity, and the potential implications of advances in this area of research for future health care.

\section{Neural plasticity research and development (R \& D)}

Over the years, questions concerning the human brain and its role in cognitive functioning have inspired generations of neuroscientists. Although even in 1987 we are far from understanding the physiology of brain and behavior, recent research findings are yielding - sometimes unexpected - insights. A number of these findings, for example, show that, contrary to traditionally held beliefs, the central nervous system is not a static network of cells merely arranging communication between organs within an organism or between the organism and its environment. The nervous system is increasingly perceived to be of a much more dynamic nature, which allows it to adapt to changing internal and external environments.

Commonly, the term neural plasticity is used to describe the adaptive capacity of the nerve cell, or neuron. It is now recognized that this adaptive capacity plays a key role in the development of specific neural networks and in the response of neurons to trauma. Furthermore, it has been shown that dynamic changes in the synapses (that is, in those places where neurons communicate with each other) may lead to totally different communication patterns, resulting in an altered output of the brain.

Following this, it is not surprising that neural plasticity is the subject of research of a variety of disciplines in the neurosciences. It can be studied at the molecular as well as at the neurophysiological level. Both morphologists and behavioral scientists have sustained interest in this area. Increasingly more clinicians have come to realize that age-related deficiencies of the nervous system, including deficiencies in cognitive functioning, may be explained by a continuous decline in neural plasticity.

In this paper we then define neural plasticity broadly: the ability of the nervous system to change its activity as a result of ongoing and previous experience or in response to injury. The following examples illustrate some recent advances in neural plasticity research.

\section{Sprouting in network development and repair}

Originally it was thought that damaged nerve cells would not survive. Yet, more and more it is becoming clear that depending on the type and place of injury neurons have the capacity to regrow their damaged cell processes. Evidence is accumulating to suggest that post-trauma neural responses are rather similar to those molecular processes that guide the organization of neuronal networks during development of the nervous system. In particular, the interplay between growing axons, the surrounding glial cells and the target tissue is of great interest in this respect.

At present, several families of so-called neurotrophic factors - i.e. factors that 
are responsible for neuronal survival and growth - can be distinguished [2]. The best studied neurotrophic factor is the nerve growth factor (NGF). Most of the well-documented effects of NGF occur in the peripheral nervous system. Evidence, however, is accumulating that NGF is also beneficial to central nervous system neurons [3]. Currently, much research is devoted to the identification, purification, and characterization of neurotrophic factors. The search is facilitated by the introduction of well-defined in vitro model systems, high-performance separation procedures, and recombinant-DNA technology. Furthermore, various cell surface components, such as cell adhesive molecules and gangliosides, and growthassociated proteins have been shown to play a role both during development and after damage $[4,5]$.

More and more scientists are focusing on the role that these trophic factors may play in pathological conditions. The first and most studied condition is the acute damage of the nervous system. However, their role in polyneuropathies, amyotrophic lateral sclerosis (ALS), Parkinson's disease and Alzheimer's disease is also being investigated. Likewise, the importance of the defective or deficient supply of trophic factors to normal aging of the peripheral and central nervous systems is currently under study. The Nobel Committee recognized the potential of this area as it awarded Levi-Montalchini and Cohen the 1986 Nobel Prize in Physiology or Medicine for their pioneering work on nerve and epidermal growth factors.

\section{Synaptic plasticity}

Modification of the brain on the basis of experience, learning, or memory is among the most essential manifestations of the plasticity of the nervous system. A matter of great interest for neurobiologists, therefore, are the plastic changes in the nervous system responsible for learning. An important line of research at present is providing evidence that learning can be described in terms of known and specific changes in the synapses of the brain [6].

The demonstration that the functional contacts between neurons, where the actual exchange of information takes place, can be modified by the activity of the nerve cells themselves, has had a dramatic impact on our thinking about the way in which neuronal networks process and preserve information. Long-lasting changes in these synaptic contacts were shown to alter the properties of the network involved [7]. A whole array of plastic changes were demonstrated via internal electrical stimuli from biochemical and morphological events to external behavioral stimuli*.

While synaptic plasticity has bearing on the neural basis of learning and memory, it may also play a significant role in understanding the deterioration in cell-

\footnotetext{
* If and when these lines of research may prove to be successful in future, the implications of a new understanding of basic premises of learning and memory for educational paradigms and systems could well be so vast that they can only be guessed at. Such implications will not be considered in the section infra dealing with policy implications, since a review of educational mechanisms on the basis of biological considerations would require a separate monograph.
} 
to-cell communication as observed in epilepsy. Recent studies have indicated that a new potential class of anti-epileptic drugs occupy the same cellular receptors that are active in the aforementioned synaptic plasticity [8].

\section{Environmental factors and neuronal plasticity}

Environmental factors play an important role in establishing and maintaining nervous system plasticity. Nutrition, drugs, environmental agents, or occupational exposure to toxic agents may influence nervous system plasticity $[9,10]$. So far, knowledge concerning the direct influence of these factors on plasticity in the adult nervous system is relatively scarce, except for their effect on plastic phenomena such as learning and their stimulation of plastic processes due to neuronal injury. In future, however, environmental factors may be found to directly influence the loss of trophic factors responsible for the maintenance of particular types of neurones [11]. The effects of these factors on the human developing brain have been studied more extensively, e.g. neurotoxic exposure may result in certain developmental disorders which involve neuronal plasticity in the brain [12].

Motor and sensory experience, including social interactions, are another type of environmental factors that affect plasticity. The importance of these factors has especially been shown by stimulation and deprivation experiments in animals [13]. For example, animal studies concerning an "enriched" environment indicate changes in anatomy and biochemistry of the brain, and subsequent behavioral changes.

\section{Technological developments based on plasticity research}

On the basis of these and other advances in plasticity research, new technological applications for nervous system disorders are appearing. In general, 3 major technological approaches can be discerned in this respect.

(1) The first approach concerns nervous tissue transplants. During the last few years transplants have been carried out with increasing frequency, especially in rodents, and have attracted considerable interest in the medical profession and the public. It has been shown, for instance, that embryonic brain tissue can be incorporated both anatomically and functionally into the adult animal central nervous system [14]. While the primary importance of neuronal transplants currently is in their use as a research tool, the success of certain transplantation experiments suggests that they might also be clinically useful. Applications have been proposed both for the neurodegenerative disease and for nervous tissue injury through trauma.

(2) Plasticity research subsequently opens possibilities for new pharmacological developments. At present, two major pharmaceutical strategies can be discerned. The first strategy involves the efficacy of general growth factors. In general, characterized growth factors, or their synthetic analogues, may provide a lead in the development of effective new drugs to treat both neurode- 
generative diseases and nervous system injury. The second strategy involved specific cell-surface components, such as cell adhesive molecules and gangliosides, which have been observed to play a prominent role in the regulation of neuronal growth in the developing and adult nervous system, both during physiological conditions and after cell damage. Gangliosides have been associated with increased survival of damaged neurons and also with sparing from secondary cell loss. Furthermore, there is evidence that gangliosides and growth factors interact, according to this model, gangliosides enhance the efficacy of growth factors [15].

(3) Finally, as environmental factors may increasingly play an important role in establishing and maintaining nervous system plasticity, interventions in both the physical environment, such as regulation of neurotoxic agents, and interventions in the social environment, such as specific behavioral programs (e.g. for the elderly) may be used to increase plasticity and thus ameliorate or enhance human health.

\section{Future implications of neural plasticity R \& D for the health sector}

From the previous it can be concluded that the areas of research and technology development included under neuroplasticity are germane to a considerable number of health-related problems. In order to illustrate this more specifically, the future applicability of above-mentioned technological approaches will now be considered for the following disease categories: Parkinson's disease, senile dementia of the Alzheimer type, and central and peripheral nerve cell trauma.

In the case of Parkinson's disease, specific neurons that produce the transmitter dopamine progressively degenerate and die. These cells are localized within the brain in the substantia nigra and project to the neostriatum. The major manifestations of Parkinson's disease are its characteristic tremor, rigidity and the inability of the patient to initiate voluntary movements. The motor and, sometimes, cognitive abnormalities accompanying the disease in a more advanced state can render a person completely disabled and ultimately, shorten life-expectancy. The estimates of prevalence vary between 60 and 180 per 100,000 in various industrialized countries (in absolute numbers, e.g. probably more than 250,000 patients only in Western Europe, with for instance 28,000 patients in the Netherlands) [16]. Despite the fact that treatment with the drug L-DOPA is generally a rather effective pharmacotherapy in terms of symptomatic treatment, unfortunately, it does not interfere with the underlying disease process. Definitive treatments or preventive measures inhibiting the degeneration of the neurons concerned, or even stimulating regeneration, still have to be found.

Currently, however, applications of neural plasticity research to Parkinson's disease are developing at a speed that would have been unimaginable to neurologists 10 years ago. Recently, the first intracerebral neural transplants in humans were performed in Sweden, Mexico and the U.S.A. with a few severely ill patients, who 
were unresponsive to L-DOPA treatment $[17,18]$. In the first clinical applications, tissue from the patient's own adrenal glands (with the ability to produce the same transmitter) was implanted close to the brain region in need of the specific transmitter. While these patients had some temporary modest improvement, it is still too early to evaluate the efficacy of these transplants. Although cell or nervous tissue transplants in localized neurodegenerative diseases, such as Parkinson's, may prove to be a viable treatment in the coming years for certain patients, well-designed clinical studies to determine efficacy and effectiveness, and cost-effectiveness would be imperative before widespread application is to be considered. Furthermore, as it becomes apparent that the use of human fetal tissue for transplantation may provide better results than using the patient's own chromaffin cells from the adrenal medulla, one is already at present confronted with complex ethical issues.

In terms of future preventive measures, recent research seems to implicate both infectious and neurotoxic agents as possible causes of Parkinson's disease by way of affecting the plasticity of the nervous system; for example, a newly identified important toxin which produces a syndrome resembling nearly all the symptoms seen in classical Parkinson's is MPTP. Both a number of chemical scientists who synthesized compounds requiring preparation of MPTP or its derivatives, and addicts who injected themselves intravenously with self-manufactured pethidine analogues including MPTP, developed Parkinson's disease [19]. The extent to which Parkinson's disease induced by insecticides, fungicides, and herbicides is also due to MPTP and/or its metabolites may well become an important area of research [20].

Ever since the interest in the "graying of our nations" took solid root in Western societies and it became clear that dementia in the elderly, in its many manifestations, was becoming a much more widespread problem than originally recognized, concern with the aging of the human brain and related disorders such as senile dementia of the Alzheimer type (SDAT) has been on the rise as well. At present, for example, approximately $7-10 \%$ of the Dutch population, 65 years of age and older, has some form of dementia [21], with SDAT accounting for about $50 \%$ of the irreversible dementias.

Worldwide, no consensus on diagnosis or disease stage classification so far has been reached [22]. The pathogenesis of SDAT is largely unknown. Neuropathological assessment of post-mortem brain material yields the presence of an extraordinary number of senile plaques and twisted filament protein tangles. Despite the fact that for some years it was thought that - in analogy to Parkinsonism - the primary cause of SDAT was a specific loss of cholinergic cells in the nucleus of Meynert, it is now evident that many parameters of neuronal and glial function are involved. It is hard to tell what are primary and what are secondary changes in the brain of the Alzheimer's patient.

Although it has been proposed that nerve cell transplants may constitute a future therapy for Alzheimer's disease [23], in view of the generalized nature of the pathology (in contrast to localized brain diseases such as Parkinson's), the poten- 
tial therapeutic use seems to be limited. Instead of transplants, other technological approaches are deemed more likely to provide effective treatment in the future. A pharmacotherapy employing neurotrophic factors should be able to delay further deterioration, provided the disease has been diagnosed in an early stage [24]. A number of major pharmaceutical companies are seeking a solution in this direction, but have not reached the stage of more extensive clinical testing as yet. A major problem which still needs to be solved before trophic factors can be effectively used in the human nervous system involves the route of administration and the resulting bioavailability of the drug at the site of the degeneration or lesion.

An alternative therapeutic approach based on plasticity research, involves the concept of an "enriched environment". Research on both healthy and demented elderly people suggests that brain and behavioral functioning can be influenced by environmental interventions, such as physical activation or, as mentioned earlier, behavioral programs [25]. Socially stimulating environments may in fact slow some of the symptoms of dementia and may delay institutionalization [26]. In general, studies on the effects of environmental interventions upon mental processes are relatively scarce; however, it is to be anticipated that this area of research may rapidly expand as insight into the mechanisms of plasticity increase. On the other hand, with new therapeutic interventions emerging for SDAT, the ability of demented persons to give informed consent needs specific consideration. Demented persons in most cases cannot adequately judge the risks involved in research, and thus lack the necessary legal capacity to provide informed consent. Ethical and legal issues center around the question who should decide whether these patients should be part of clinical trials, and what the standards should be for ensuring protection of their rights, thus to an extent constraining the practical possibilities for the expansion concerned.

Accidents involving central or peripheral nerve trauma account for a large number of deaths and disabilities each year. Many survivors of brain and spinal cord damage continue to have some form of disability $[27,28]$. Since most victims are below 35 years of age, the long-term costs of their care are often enormous. Injury involving peripheral nerves regularly results in lasting deficiencies; although peripheral nerves may display some regenerative outgrowth, functional recovery is often poor.

Although some progress is made in our understanding of what adaptive capacities the brain has and how to mobilize them, it is highly questionable if nerve cell transplants will become an effective approach in the coming years to treat those who have suffered from brain damage due to accidents. More results in this respect can be expected from pharmacological treatments, e.g. intracerebral injections of growth factors have been shown to enhance neuronal survival, and injections of purified gangliosides seem to reduce several behavioral deficiencies $[29,30]$. With regard to spinal cord injuries, various transplantation strategies may be used in future to restore function below the lesion. Research on so-called "locomotiongenerators" (groups of neurons in the spinal cord essential to locomotion) and efforts towards constructing an unbroken pathway from the site of the lesion (pass- 
ing or bridging the area of the lesion itself) to the target muscle may lead to practical clinical applicability [31]. The prospects for peripheral nervous system trauma are more promising in the short term. Good clinical functional results have been obtained using autologous nerve grafting techniques. In addition to nerve tissue, 'polymers' are now being used to 'bridge' gaps and stimulate regeneration of nerve fibers. Much work is furthermore directed towards biochemically enhancing the regeneration of peripheral nerves; local administration of trophic factors, for instance melanocortins, at the site of the nerve damage by means of new drug delivery systems remarkably enhances peripheral outgrowth and subsequent recovery [32].

Having considered the potential clinical applicability of developments in plasticity research for a limited number of disease categories, let us now turn to overall policy issues pertaining to the area of plasticity research and development.

\section{Policy conclusions}

Present-day neurosciences research in general and plasticity research in particular is at an exciting stage of its development with new scientific findings appearing at a rapid pace. The momentum that we observed in the previous paragraphs promises to result in new technological applications in the years to come. In this paper 3 major technological approaches have been discerned in this respect: nerve tissue transplants, pharmacological developments, and environmental interventions. What then is the relevance of these future interventions for health care policy making today?

It was shown that impairments in plasticity are pertinent to a considerable number of nervous system disorders. Whereas cardiovascular diseases and cancers still dominate present-day mortality pattens in most industrialized countries, morbidity and functional disability measures increasingly indicate the importance of nervous system disorders. For example, for a Western country like the Netherlands mental diseases accounted for $23.5 \%$ and muscular-skeletal diseases for $22.8 \%$ of the 1983 disability pensions, while cardiovascular diseases accounted for $10.9 \%$ and cancers for $1.8 \%$ of total pension disbursements in that country [33]. Trendwise, extrapolations would show that neurological, behavioral, and communicative disorders may gain significantly in importance in the 1990s and beyond. Hypothetically speaking, it would not even be beyond the imagination to postulate that the large question-mark governing the guestimates which (combination of) disease patterns would be the successors to cardiovascular diseases and cancers beyond the 1990s, may well be answered by a disproportionate relevance, possibly even a dramatic priority-gaining of the neurological, behavioral and communicative disorders in the first decennia of the next millenium.

At this moment our health care system would not be geared to respond in any way to such a shift in priorities. An effective health care policy response needs to take into account the possibility and dimensions of such a shift; insight therefore is required in changing structures of health needs and demands on the one hand 
and the probabilities of future effective health care interventions on the other [34].

This paper is concerned with the probabilities of improvements in prevention and treatment of certain nervous system disorders following from plasticity research. Given the consistently rapid pace of progress, we conclude that the probability of such new technological applications emerging for injuries involving nerve tissue trauma and Parkinson's disease in the coming decade is high. However, if no sudden breakthroughs occur, widespread clinical application for a complex disease category such as Alzheimer's disease may well take another 10 years. The moment that such new clinical applications emerge, however, large-scale implications for the delivery of health care and concomitant expenditures can be expected. For instance, if the application of results of plasticity research would delay the institutionalization because of SDAT for 5 years, rough calculations indicate that the number of demented persons in Dutch nursing homes would decrease by $26 \%$ in the year 2000, compared with a no-change scenario [35]. In view of longterm depreciation periods, these kinds of implications need to be taken into account in present-day planning of and investments in health care facilities, such as hospitals, long-term care institutions or their future alternatives. Furthermore, the consequences of the aforementioned developments for health care manpower planning and for overall health care expenditures need to be considered as well, including both their potential to decrease costs - as with certain preventive measures - and increase costs. With regard to the latter, the issue of recurrent expenditures of treatments over longer periods of time is of importance, e.g. the costs of follow-up treatments (possibly over a period of 50 years) of a 20-year-old trauma patient receiving nerve tissue transplants. Inattention to these kinds of future developments and their various degrees of probability could well result in serious "losses" for the health sector, thus underscoring the need to take future alternatives into account and adjust health policy plans accordingly. In view of the importance of nervous system disorders, health care policy makers may - in addition - decide to try and close the gap from research findings towards clinical application more rapidly, by assuring adequate access to technological, financial, and human resources.

In conclusion, above-mentioned considerations seem straightforward issues of management and strategic planning in view of new data and new priorities, i.e. adapting the present system to future probabilities. One major caveat must be put forward, however. The warning would concern the social, ethical and judicial aspects surrounding future technological applications in the area of nervous system plasticity. Planning and management into the 1990s of disease categories involving the most sensitive of human functions should not be confused with the planning and management of, e.g. a material good like electronic mail into the 1990s. If health policy is to make itself familiar with this new field - a purpose to which this paper hopes to contribute - and is to react sensibly to the new probabilities outlined above, it would do well to actually initiate a timely debate on such social and ethical issues itself. 


\section{References}

1 Steering Committee on Future Health Scenarios. Developments in the Regeneration, Repair, and Reorganization of nervous tissue and their applications to Alzheimer's disease, Parkinson's disease, and accidents involving nervous system trauma, The Hague, March 1987 (in print).

2 Varon, S., Factors promoting the growth of the nervous system, Neurosciences, 3 (1985) 62.

3 Marx, J.L., Nerve growth factor acts in brain, Science, 232 (1986) 1341-1342.

4 Rutishauer, U., Molecular and biological properties of a neural cell adhesion molecule, Symposium on Quantitative Biology, 48 (1983) 501-514.

5 Karpiak, S., Vilim, F. and Mahadik, S.P., Ganglioside effects on neuronal plasticity during developing, Neurotoxicology, 7 (1986) 350.

6 Kendal, B. and Schwartz, J.H., Principles of Neuro-Sciences, 2nd edn., Elsevier, Amsterdam, 1985.

7 Morris, R.G.M., Hagan, J.J. and Rawlins, J.N.P., Allocentric spatial learning by hippocampectomized rats: a further test of the "spatial mapping" and "working memory" theories of hippocampal function, Quarterly Journal of Experimental Psychology, Section B - Comparative and Physiological Psychology, 38 (1986) 365-396.

8 Lopes da Silva, F.H., Hippocampal kindling: physiological evidence for progressive disinhibition. In Wolf, P. and Janz, C.E. (eds.), Advances in Epileptology, Vol. 16, Raven, New York, 1986, Ch. 12.

9 Calne, D.B., McGeer, E., Eisen, A. and Spencer, P., Alzheimer's disease, Parkinson's disease, and motoneurone disease: abiotropic interaction between aging and environment? The Lancet, 2 (1986) 1067-1070.

10 Garruto, R.M., Swyt, C., Yanagihara, R., Fiori, C.E. and Gajdusek, D.C., Intraneuronal co-localization of silicon with calcium and aluminum in amyotrophic lateral sclerosis and parkinsonism with dementia of Guam, The New England Journal of Medicine, 315 (1986) 711.

11 Bower, B., Tracking the roots of Parkinson's disease, Science News, 128 (1985) 212.

12 Gezondheidsraad, Advies inzake teratogeniteit van chemische stoffen, The Hague, The Netherlands, 1985.

13 Mirmiran, M., van Gool, W.A., van Haaren, F. and Polak, C.E., Environmental influences on brain and behavior in aging and senile dementia, Progress in Brain Research, 70 (1986) 443-460.

14 Redmond, D.E., Roth, R.H., Elsworth, J.D., Sladek, J.R., Collier, T.U.K., Deutch, A.U. and Haber, S., Fetal neuronal grafts in monkeys given methylphenyltetrahydropyrdine, The Lancet, 1 (1986) 1125-1127.

15 Toffano, G., Savoini, G., Aldinio, C., Valenti, G., Dal-Taso, R., Leon, A., Calza, L., Zini, J., Agnati, L.S. and Fuchs, K. Effects of gangliosides on the functional recovery of damaged brain, Advances in Experimental Medical Biology, 174 (1984) 475-488.

16 Canadian Journal of Neurological Sciences, 1984, Supplement 11.7, pp. 89-237.

17 Bjorklund, A. and Stefenevi, U., Intracerebral neural implants: neuronal replacement and reconstruction of damaged circuitries, Annual Review on Neurosciences, 7 (1984) 279-308.

18 Madrazo, I., Drucker-Colin, R., Diaz, V., Martinez-Mata, J., Torres, C. and Becerril, J.J., Open microsurgical autograft of adrenal medulla to the right caudate nucleus in two patients with intractable Parkinson's disease, New England Journal of Medicine, 316 (1987) 831-834.

19 Langston, J.W., Ballard, P., Tetrud, J.W. and Irwin, I., Chronic Parkisonism in humans due to a product of meperidine - analog synthesis, Science, 219 (1983) 979-980.

20 Bocchetta, A. and Corsini, G.U., Parkinson's disease and pesticides, The Lancet, 2 (1986) 2199-2205.

22 Traber, J. and Gispen, W.H. (Eds.), Senile Dementia of the Alzheimer's type, Early Diagnosis, Neuropathology and Animal Models, Springer, Berlin-Heidelberg, 1985.

23 Gash, D.M., Collier, T.J. and Sladek, J.R., Neural transplantation: a review of recent developments and potential applications to the aged brain, Neurobiology of Aging, 6 (1985) 131-150.

24 Appel, S.H., Ojika, K., Tomozawa, Y. and Bostwick, R., Trophic factors in brain aging and disease. In Traber, J. and Gispen, W.H. (Eds.), Senile Dementia of the Alzheimer's Type, Early Diagnosis, Neuropathology and Animal Models, Springer, Berlin-Heidelberg, 1985, pp. 218-230.

25 Charatan, F.B., Mental stimulation and deprivation as role factors in Senility. In Rotschild, H. (Ed.), Risk Factors for Senility, Oxford University Press, Oxford, 1984. 
26 Swaab, D.F. and Fliers, E., Clinical strategies in the treatment of Alzheimer's disease, Netherlands Institute for Brain Research, 1985.

27 Kraus, J.F., Black, M.A., Hessol, N., Ley, P., Rokaw, W., Sullivan, C., Bowers, S., Knowlton, S. and Marshall, L., The incidence of acute brain injury and serious impairment in a defined population. American Journal of Epidemiology, 119 (1984) 186-201.

28 Fine, P.R., Kuhlemeier, H.U., De Vivo, M.J. and Stover, S.L., Spinal cord injury: an epidemiologic perspective, Paraplegia, 17 (1979-1980) 237-250.

29 Cotman, C.W. and Nieto-Sampedro, M., Progress in facilitating the recovery of function after central nervous system trauma, Annals of the New York Academy of Sciences, 181 (1985) 83-104.

30 Sabel, B.A. and Stein, D.G., Pharmacological treatment of central nervous system injury, Nahere, 323 (1986) 493.

31 Reier, P.J., Neural tissue grafts and repair of the injured spinal cord, Neuropathology and Applied Neurobiology, 11 (1985) 81-104.

32 Edwards, P.M., Kuiters, R.R.F., Boer, G.J. and Gispen, W.H., Recovery from peripheral nerve transection is accelerated by local application of $\alpha-\mathrm{MSH}$ by means of microporous accurel polypropylene tubes, Journal of the Neurological Sciences, 76 (1986) 171-176.

33 Anticipating and Assessing Health Care Technology, Volume I, General Considerations and Policy Conclusions, Kluwer, Dordrecht, 1987.

34 Pannenborg, C.O., Contraceptive needs in the Third World: the present paradox of a future family planning-technology nexus. In van Keep, P.A., Ellison D. and de Wied, D. (Eds.), Contraceptives in the year 2001, Excerpta Medica, Amsterdam, 1987, pp. 21-44.

35 Stuurgroep Toekomst Scenarios Gezondheidszorg (STG), Ouder worden in de toekomst. Scenarios over gezondheid en vergrijzing 1986-2000, The Hague, 1986. 